\title{
Effect of Particle Size and Natural Organic Matter on the Migration of Nano- and Micro-Scale Latex Particles in Saturated Porous Media
}

Journal of Colloid and Interface Science

Revised January 19, 2008

ANDREW J. PELLEY and NATHALIE TUFENKJI*

Department of Chemical Engineering, McGill University, Montreal, Quebec H3A 2B2, Canada

${ }^{*}$ Corresponding Author. Phone: (514) 398-2999; Fax: (514) 398-6678; E-mail: nathalie.tufenkji@mcgill.ca 


\begin{abstract}
In the interest of fully assessing the potential environmental risks linked to "nano-litter", we need to be able to predict the persistence, toxicity, and mobility of engineered nanomaterials in the natural subsurface environment. To examine the effects of particle size and natural organic matter on nanoparticle mobility, laboratory-scale filtration experiments were performed using different sized model nanomaterials (i.e., latex colloids having diameters of 50,110, and $1500 \mathrm{~nm}$ ) in the presence and absence of $5.0 \mathrm{mg} / \mathrm{L}$ Suwannee River Humic Acid (SRHA). At low ionic strengths (1 - $10 \mathrm{mM}$ $\mathrm{KCl}$ ), an increase in attachment efficiency $(\alpha)$ with increasing particle size was observed. This result contrasts with predictions of particle filtration based on attachment in the primary energy minimum of the particle-grain interaction energy profile evaluated using Derjaguin-Landau-Verwey-Overbeek (DLVO) theory. The presence of SRHA generally resulted in a decrease in $\alpha$ over the range of experimental conditions investigated. Results of particle transport experiments combined with particle characterization measurements suggest that the decrease in colloid attachment in the presence of SRHA is related to the combined influence of the mechanisms of charge stabilization and steric stabilization.
\end{abstract}

Keywords: nanoparticle, filtration, groundwater, contamination, humic acid, steric stabilization, particle size, zeta potential. 


\section{Introduction}

Global activities involving nanotechnologies are estimated at US\$45 billion [1] and in Québec alone, more than $\$ 400$ million has already been invested in nanotechnology in an effort to promote research in this area. Nanomaterials are used in the fabrication of paints, coatings, cosmetics, and many other consumer goods. It is anticipated that by 2015 , the global market for "nano" products will exceed US\$1 trillion, thereby affecting several economic sectors [2, 3]. Although nanotechnologies promise many social and economic benefits, recent reports published in North America and Europe underline the potential environmental and public health risks linked to this "nano" revolution [1]. A portion of manufactured nanomaterials will end up in the natural environment (e.g., as a result of accidental spills or released in industrial wastewaters). A wide range of manufactured nanoparticles have been shown to exhibit properties of increased reactivity and toxicity $[1,4-6]$.

The impact of manufactured nanomaterials on the environment and human health will depend on their fate in natural systems. Despite the risks associated with the release of nanomaterials in natural soils and groundwaters, few studies have addressed their behavior in these systems. The few reported studies on the transport and fate of engineered nanomaterials in granular matrices have been conducted using laboratory-scale experimental systems [7-11]. In most cases, chromatography columns packed with glass beads have been used to study the filtration of various nanomaterials suspended in simple electrolytes. However, the natural subsurface environment exhibits significant physical, biological, and geochemical heterogeneities, including the presence of biofilms, natural organic matter (NOM), and changing groundwater and sediment chemistry that can influence the degree of nanoparticle migration in granular matrices. For instance, some researchers have demonstrated that adsorption of NOM onto suspended colloids and/or sediment grain surfaces generally facilitates colloid transport in the subsurface [12- 
14]; yet, little is known about its impact on the stability and migration potential of different-sized nanoparticles in groundwater environments.

Initial investigations into the mobility of nanomaterials in systems that model groundwater aquifers have shown that varying the surface functionalization of nanoparticles can lead to significant differences in their migration potential $[7,11]$. For example, nanoparticulate fullerene aggregates $\left(n \mathrm{C}_{60}\right)$ have very limited mobility in porous media due to their low solubility in water while fullerol has been found to be highly mobile [11]. Findings such as these underscore the need to avoid using generalizations in the implementation of a regulatory environmental framework for protection of groundwater supplies from contamination by engineered nanomaterials. Rather, it is necessary to obtain a better mechanistic understanding of the key factors controlling nanomaterial migration over a broad range of environmentally relevant physicochemical and geochemical conditions.

Particles suspended in groundwater will contact the surface of a sediment grain (collector) as a result of three governing mechanisms: (i) interception, (ii) gravitational sedimentation, and (iii) Brownian diffusion $[15,16]$. The latter is of particular importance in the retention of nanoparticles as their high diffusivity leads to a higher incidence of collisions with the surface of soil grains. Classical colloid filtration theory takes into account the contribution of these three transport mechanisms to particle retention in a parameter referred to as the single-collector contact efficiency $\left(\eta_{0}\right)[15,17]$. However, simply making contact with a collector surface does not ensure that a particle will attach to the grain surface as various colloidal interactions and forces (e.g., electrical double layer repulsion) may prevent particle removal from the pore fluid. Hence, the actual single-collector removal efficiency $(\eta)$ is expressed as the product of an empirical attachment efficiency $(\alpha)$ and the single-collector contact efficiency $\left(\eta_{0}\right)$. The attachment 
efficiency represents the fraction of collisions between suspended particles and collectors that result in attachment. Currently, there are no satisfactory theoretical models to predict the attachment efficiency under conditions deemed unfavourable for particle attachment; namely in the presence of repulsive Derjaguin-Landau-Verwey-Overbeek (DLVO) interactions [18, 19]. Attempts have been made to incorporate the classic DLVO theory into the convective-diffusion equation in an effort to predict particle deposition rates [16, 20, 21]. In general, though, experimentally determined particle deposition rates are orders of magnitude greater than theoretical values. Furthermore, results of particle transport and deposition experiments are often not in agreement with qualitative predictions based on DLVO theory [22-24]. For instance, some studies indicate that particle retention in granular porous media increases with particle size [2325], whereas others have shown that particle attachment efficiency may be independent of particle size $[22,26]$. In contrast, consideration of irreversible particle deposition in the primary energy minimum of the DLVO interaction energy profile predicts a decrease in particle deposition rates with increasing particle size (under unfavourable conditions for deposition) [16]. Various explanations have been proposed to rationalize these discrepancies such as the surface roughness of the particles or collectors, hydrodynamic interactions, the non-homogeneity of surface charge distribution, the dynamics of colloidal interactions, physical straining or trapping of particles, and deposition in the secondary energy minimum of the DLVO interaction energy profile [22-25]. While each of these factors likely merit attention, the current understanding of these factors precludes their inclusion in any rigorous quantitative assessment.

The general objective of this paper is to determine the effect of particle size in conjunction with that of the presence of NOM on the transport of nanomaterials in a model groundwater system. Laboratory-scale packed column experiments were used to examine the deposition 
behaviour of model latex colloids over a broad range of solution ionic strengths (IS). Sulphate latex particles ranging in size from $50 \mathrm{~nm}$ to $1500 \mathrm{~nm}$ were selected as model anthropogenic nanomaterials for the transport studies revealing an increase in colloid retention with increasing particle size. In a second series of packed column experiments, the effect of NOM on the transport and retention of micron and nano-sized colloids was investigated by including Suwannee River Humic Acid (SRHA; $5 \mathrm{mg} / \mathrm{L}$ ) in the artificial groundwater solution. The use of different-sized colloids of similar surface chemistry allows us to examine whether the presence of NOM similarly impacts the transport and fate of nanoparticles as it does that of micron-scale colloids. Physicochemical properties of the particles characterized over the range of solution conditions investigated are used in the interpretation of data obtained from the packed column experiments.

\section{Materials and Methods}

\subsection{Model Colloids, Natural Organic Matter and Granular Media}

Three different-sized $(50 \mathrm{~nm}, 110 \mathrm{~nm}$, and $1500 \mathrm{~nm})$ surfactant-free polystyrene latex microspheres with sulphate functional groups were used as model colloids (Interfacial Dynamics Corporation, Portland, OR). These particles were selected for investigation because they range in size from nano-scale to micron-scale while having comparable surface charge densities $(0.9$ $\mu \mathrm{C} / \mathrm{cm}^{2}$ ). Colloid suspensions used in colloid filtration experiments were prepared by diluting stock colloidal samples in electrolyte of varying ionic strength $(1-100 \mathrm{mM} \mathrm{KCl}$ in deionized MilliQ water) at an unadjusted $\mathrm{pH}$ of $5.7 \pm 0.2$.

Suwannee River Humic Acid (SRHA) was used as a representative organic matter

(International Humic Substances Society). Humic substances represent a significant fraction of 
organic carbon present in aquatic systems [27] and are found in natural waters at concentrations of $0.1-200 \mathrm{mg} / \mathrm{L}$ dissolved organic carbon (DOC) [28]. In colloid deposition experiments conducted with NOM, SRHA was applied at a concentration of $5 \mathrm{mg} \mathrm{NOM} / \mathrm{L}$. Electrolyte solutions were adjusted to $\mathrm{pH} 5.7 \pm 0.2$ using $0.1 \mathrm{M} \mathrm{NaOH}$.

High purity quartz sand (Sigma-Aldrich) was selected as a model granular material to allow careful control of the experimental system. Although not perfectly representative of natural soils, the pure quartz sand allows us to interpret the experimental results within the context of the DLVO theory of colloidal stability. Prior to use, the sand was sieved with Nylon sieves (U.S. standard mesh sizes 50 and 70, 300 and $212 \mu$ m openings, respectively) to obtain a sand sample having an average grain diameter of $256 \mu \mathrm{m}$. The sand was cleaned according to the procedure outlined by Litton and Olson [29]. Briefly, this involves first soaking the sand in $12 \mathrm{M} \mathrm{HCl}$ for $24 \mathrm{hrs}$, then washing with DI water until the $\mathrm{pH}$ of the rinse solution reaches $\sim 5.6$. The media was then baked in a furnace (MAS 7000 Microwave Furnace) at $120^{\circ} \mathrm{C}$ for $1 \mathrm{hr}$ and then at $800^{\circ} \mathrm{C}$ for $5 \mathrm{hrs}$. This process is designed to ensure that the media is not only dry but also to remove any metal or organic impurities from the sand surface.

\subsection{Characterization of Particles}

The hydrodynamic diameter of the three different-sized latex colloids was verified over the range of solution chemistries investigated (in the presence and absence of SRHA) by dynamic light scattering (DLS, ZetaSizer Nano, Malvern). Colloid suspensions prepared in the electrolyte solution of interest $(1-100 \mathrm{mM} \mathrm{KCl}$ in DI water at $\mathrm{pH} 5.7 \pm 0.2)$ were placed in a disposable $1-\mathrm{cm}$ polystyrene cuvette for measurement. Each measurement was repeated using at least three different samples. 
Laser Doppler Velocimetry in conjunction with Phase Analysis Light Scattering (ZetaSizer Nano, Malvern) was used to characterize the electrokinetic properties of the latex particles for each solution condition examined. Electrophoretic mobility was measured at $25^{\circ} \mathrm{C}\left( \pm 1{ }^{\circ} \mathrm{C}\right)$ using particle suspensions $\left(1.2 \times 10^{11}, 1.2 \times 10^{10}\right.$ and $8.0 \times 10^{6}$ particles $/ \mathrm{mL}$, for the 50,110 , and 1500 $\mathrm{nm}$ particles, respectively) prepared in the same electrolyte solutions as described above. Each measurement was repeated using at least three different samples. Electrophoretic mobilities were converted to zeta potential using the Smoluchowski equation [30].

\subsection{Colloid Filtration Studies}

The transport and deposition of the three different-sized colloids was examined separately using a laboratory-scale glass column packed with the clean quartz sand. Prior to packing the glass column, the granular media was preconditioned for a period of $24 \mathrm{hrs}$ by soaking in the electrolyte solution (on a shaking platform) to be used in the experiment. The conditioned granular material was then wet-packed into the clean glass column $(15-16.5 \mathrm{~cm}$ packed length, $1 \mathrm{~cm}$ internal diameter) to avoid air entrapment using vibration to ensure uniform packing and to minimize any possible layering of the sand. The packed bed had a porosity of 0.38 . Next, at least twenty pore volumes of the electrolyte solution of interest were passed through the column to ensure that the column was fully equilibrated with this solution (one pore volume is the volume of free-space in the column unoccupied by media). Following equilibration, approximately three pore-volumes of the colloid suspension (also preconditioned for $24 \mathrm{hrs}$ by soaking in the electrolyte solution of interest) was injected into the packed column (constant approach velocity of $2.12 \times 10^{-2} \mathrm{~cm} / \mathrm{s}$ ) and the effluent colloid concentration was monitored using a UV-Vis spectrophotometer (HewlettPackard Model 8453) at a wavelength of $254 \mathrm{~nm}$ and using a 1-cm flow-through cell. The 
concentration of the influent colloid solution $\left(C_{0}\right)$ was the same as that used for characterization of particles (section 2.2.) and was obtained by measuring the absorbance of the suspension when bypassing the column. Each colloid deposition experiment was repeated at least twice. All particle suspensions used in colloid filtration studies were prepared in the same manner as those used to determine particle size and electrophoretic mobility (using the ZetaSizer Nano, section 2.2.). The particles were preconditioned for $24 \mathrm{hr}$ in the electrolyte solution (in the presence or absence of SRHA) to be used in a given experiment.

\section{Results and Discussion}

\subsection{Colloid Properties}

A series of experiments were performed to characterize the materials used in the investigation to aid in the interpretation of data obtained from the colloid deposition studies. These measurements include particle sizing using dynamic light scattering and the characterization of surface (zeta) potential from measurements of electrophoretic mobility. Results of these characterizations and a discussion of how they can aid in the interpretation of colloid filtration experiments are presented below.

Figure 1 shows the average hydrodynamic diameter of the three sulphate latex colloids measured in the absence and presence of SRHA $(5 \mathrm{mg} / \mathrm{L})$ over a range of solution ionic strengths $(1-100 \mathrm{mM} \mathrm{KCl})$ at $\mathrm{pH} 5.7 \pm 0.2$. The data generally confirms the sizing information provided by the manufacturer of the latex colloids and demonstrates that particle coagulation or flocculation is not taking place. While some slight deviation between the measured hydrodynamic diameter of 
the particles and the size quoted by the manufacturer may be observed, the results in Figure 1 show that the hydrodynamic diameter was generally unchanged in the presence of SRHA.

[FIGURE 1]

Measurements of particle electrophoretic mobility were obtained using colloid suspensions prepared in $\mathrm{KCl}$ in the absence and presence of $5 \mathrm{mg} / \mathrm{L}$ SRHA. Mobilities are converted into zeta potential using the Smoluchowski approach and these are presented in Figure 2. All three latex colloids are negatively charged over the range of solution conditions investigated. The electrical charge and potential of latex particles originates from the dissociation of functionalized surface groups. In the electrostatic double-layer model, charged surfaces in aqueous solution attract counter-ions from the solution. The presence of counter-ions effectively "masks" the charge on the particle's surface. As the ionic strength of the solution is increased (at constant $\mathrm{pH}$ ), compression of the electrical double layer gives rise to a continuous decrease in the magnitude of the surface (zeta) potential [31]. However, inspection of Figure 2 reveals that rather than continuously decreasing with increasing ionic strength, the general shape of two of the three sets of measurements exhibit a maximum at a moderate ionic strength $(10 \mathrm{mM} \mathrm{KCl}$ and $3 \mathrm{mM} \mathrm{KCl}$ for the 1500 and $110 \mathrm{~nm}$ particles, respectively).

[FIGURE 2]

Various qualitative explanations have been proposed for the behaviour noted in Figures $2 \mathrm{~b}$ and 2c including the preferential adsorption model and the so-called hairy layer model [32] The preferential adsorption model argues that this maximum may be explained by the preferential 
adsorption of co-ions onto the surfaces of latex particles amplifying the electrokinetic potential of the colloids $[33,34]$. The hairy layer model envisions the surface of polymer lattices as being comprised of polyelectrolyte chains carrying the surface charge [35]. As ionic strength is varied, this layer is hypothesized to expand and contract due to the repulsion of the functional groups. As this occurs, the location of the plane of shear is affected and consequently so too is the electrokinetic potential. Elimelech and O'Melia considered both of these models and while they do not argue against the existence of a hairy layer at the surface of latex particles, they concluded that the maximum that often occurs in the mobility curves of negatively charged polystyrene latex particles is more likely to be determined by the combined action of co-ions and counter-ions at the interface [32]. Others have shown that the standard electrokinetic model predicts the observed maxima in measured electrophoretic mobility [36]. Furthermore, this latter study demonstrates how the maximum becomes increasingly important with increasing particle size (as observed in Figure 2) [36].

The presence of SRHA leads to an increase in the absolute zeta potential of the colloid suspensions for the two smaller sized colloids (i.e., $50 \mathrm{~nm}$ and $110 \mathrm{~nm}$ ). Several other researchers have shown that dissolved NOM can impart a negative surface charge to suspended particles, including latex microspheres, hematite particles, and microorganisms such as Cryptosporidium parvum oocysts $[12,14,37,38]$. However, for the case of the $1500 \mathrm{~nm}$ colloids, the presence of NOM leads to an unusual decrease in the absolute value of zeta potential as can be observed in Figure 2c.

Careful inspection of the absolute values of zeta potential for the $50 \mathrm{~nm}$ and $110 \mathrm{~nm}$ particles relative to those of the $1500 \mathrm{~nm}$ particles yields a possible explanation for the reversal in charge behaviour that was observed in the presence of SRHA for the $1500 \mathrm{~nm}$ colloids. Since the 
SRHA bears a particular average charge of its own, then rather than amplifying or neutralizing the charge of the particles, the SRHA could be "masking" the charge of the particles with its own. The data shown in Figure 2 shows that the average zeta potential of the $1500 \mathrm{~nm}$ colloids is considerably higher than that of either of the two smaller nanoparticles. It is possible that the average charge borne by the NOM could lie somewhere in between that of the $1500 \mathrm{~nm}$ particles and the two smaller sized colloids. If so, it is possible that the reversal in charge behaviour observed for the larger colloids in the presence of SRHA can be explained by the average potential of the SRHA lying somewhere in between that of the smaller and larger latex particles. Moreover, as the particle concentration of the $1500 \mathrm{~nm}$ colloids is considerably lower than that used for the two smaller colloids, the available surface area for sorption of SRHA on the $1500 \mathrm{~nm}$ colloids is significantly lower than for the $50 \mathrm{~nm}$ and $110 \mathrm{~nm}$ colloids. Hence, if similar masses of SRHA are sorbing onto the particle surfaces, the surface coverage of the $1500 \mathrm{~nm}$ colloid may be much higher and therefore better able to mask the underlying particle charge. Davis [39] previously showed that that the electrophoretic mobility of alumina particles became more negative with increasing NOM surface coverage.

\subsection{Colloid Filtration Studies}

Two series of colloid filtration experiments were conducted with all three sulphate latex particles: the first series was conducted using simple monovalent electrolyte solutions $(1-100 \mathrm{mM} \mathrm{KCl})$, and in the second series, $5 \mathrm{mg} / \mathrm{L}$ SRHA was added to electrolyte solutions. Figure 3 shows representative particle breakthrough curves for experiments conducted with $1500 \mathrm{~nm}$ particles in the absence of SRHA where the normalized particle concentration at the column effluent $\left(C / C_{0}\right)$ is 
plotted as a function of pore volumes (dimensionless time). The data shows a decrease in the breakthrough particle concentration at the column effluent (i.e., an increased retention of the particles) with increasing solution ionic strength.

\section{[FIGURE 3]}

To quantitatively compare the colloid transport behaviour under different experimental conditions (i.e., for different particle sizes and in the presence and absence of SRHA), the attachment efficiency was calculated using colloid filtration theory (CFT) as follows [15]:

$$
\alpha=-\frac{2}{3} \frac{d_{\mathrm{c}}}{(1-\varepsilon) L \eta_{0}} \ln \left(C / C_{0}\right)
$$

where $d_{\mathrm{c}}$ is the diameter of the sand grains, $\varepsilon$ is the bed porosity and $L$ is the bed length. The value of $C / C_{0}$ in eq 1 was obtained from each particle breakthrough curve by averaging values measured between pore volumes 1.9 and 2.1 (i.e., under clean-bed conditions). Values of $\eta_{0}$ for each experimental condition were determined using a correlation developed by Tufenkji and Elimelech [17]. Figure 4 summarizes the calculated values of attachment efficiency for each of the three latex particles obtained in the absence of NOM.

\section{[FIGURE 4]}

\subsubsection{Role of Salt Concentration}

Inspection of Figure 4 reveals insight into the transport and filtration behaviour of the three different-sized model colloids suspended in simple monovalent electrolyte solutions. At low salt concentrations $(1-10 \mathrm{mM} \mathrm{KCl})$, as ionic strength of the electrolyte was increased, attachment efficiency generally increased for all particles examined. The particle zeta potential data presented 
previously (Figure 2) also shows that the potential generally becomes less negative with increasing salt concentration. Hence, the particle attachment behaviour observed here is generally in qualitative agreement with the DLVO theory of colloidal stability whereby the extent of physicochemical attachment increases with decreasing absolute zeta potential $[18,19]$. It is interesting to note that the notable drop in $\alpha$ for the $1500 \mathrm{~nm}$ colloid at $10 \mathrm{mM}$ IS is corroborated by a corresponding increase in absolute zeta potential at the same IS (Figure 2). At higher IS (10 $-100 \mathrm{mM} \mathrm{KCl}$ ), the effect of increasing salt concentration on $\alpha$ is lessened. Interestingly, even at the highest ionic strength examined $(100 \mathrm{mM} \mathrm{KCl})$, the attachment efficiency does not reach the theoretical maximum of 1 .

To interpret the particle deposition trends observed in the present study, the DLVO theory is used to calculate colloid-surface interaction energy profiles $[18,19]$. The DLVO theory considers the sum of London-van der Waals (VDW) attraction and electrostatic double-layer repulsion $(\mathrm{EDL})$. The total interaction energy $\left(\Phi_{\mathrm{T}}\right)$, namely the sum of VDW and EDL interactions, was determined by considering the particle-quartz grain system as a sphere-plate interaction. Theoretical expressions proposed by Gregory were used to calculate VDW interactions accounting for the effect of retardation where a Hamaker constant of $1 \times 10^{-20} \mathrm{~J}$ was selected for the polystyrene-water-quartz system [40]. Constant-potential EDL interactions were calculated using the expression of Hogg, Healy and Fuerstenau [41] where the zeta potentials of the latex colloids and the quartz sand were used in place of the respective surface potentials. The values of zeta potential used for the collectors (quartz sand) were taken from Redman et al [42] as they were obtained at the same $\mathrm{pH}$ and ionic strengths used in this study. Calculated total interaction energies for the simple electrolyte system are plotted as a function of separation 
distance in Figures $5 \mathrm{a}, \mathrm{b}$, and c, for the $50 \mathrm{~nm}, 110 \mathrm{~nm}$, and $1500 \mathrm{~nm}$ colloids, respectively. The data is plotted in terms of $k_{\mathrm{B}} T$, where $k_{\mathrm{B}}$ is the Boltzmann constant and $T$ is absolute temperature.

\section{[FIGURE 5]}

At large separation distances (i.e., greater than $50 \mathrm{~nm}$ ), the latex colloids do not "feel" the silica surface. As a particle approaches the quartz grain, it first experiences a weak attractive force (described as the secondary energy minimum). Upon closer approach, a substantial repulsive energy barrier is encountered that increases in height with decreasing solution ionic strength. The predicted height of the energy barrier $\left(\Phi_{\mathrm{B}}\right)$ ranges from 420 to $7200 k_{\mathrm{B}} T$ for the largest latex colloid, but only from 2 to $130 k_{\mathrm{B}} T$ for the smallest nanoparticle. These DLVO calculations suggest that the two larger colloids (110 and $1500 \mathrm{~nm}$ ) should experience considerable repulsive force upon approach to the grain surface and are not expected to attach to the grain surface (i.e., to deposit in the primary energy well located behind the repulsive energy barrier). Alternatively, the depth of the secondary energy minimum $\left(\Phi_{\min }\right)$ increases with increasing IS and, hence, the observed increase in $\alpha$ values with salt concentration could be attributed to deposition in this barrierless energy well.

\subsubsection{Role of Particle Size}

DLVO calculations presented in Figure 5 demonstrate how the particle-surface interaction energy profiles are very sensitive to changes in particle size. Specifically, the key attributes of these profiles, namely, the height of the repulsive energy barrier and the depth of the secondary energy well, both increase in magnitude with increasing particle diameter. Hence, DLVO theory predicts 
a marked effect of particle size on particle attachment efficiency. Yet, an earlier study examining the effect of particle size on colloid transport in saturated porous media revealed no differences in the deposition kinetics of different sized latex particles $(46-753 \mathrm{~nm})$ [22]. These authors attributed the observed failure of DLVO theory to the influence of dynamics of interaction - i.e., the coupling of electrodynamics and hydrodynamics [22]. In contrast, a more recent study [24], revealed significant differences in particle deposition rates for different sized latex colloids. The observed variation in particle attachment behavior was attributed to deposition in the secondary energy well, which becomes more significant with increasing particle size. Although this latter study [24] provides insight into the mechanisms controlling particle deposition in granular porous media, these experiments were limited to latex particles greater than $245 \mathrm{~nm}$ in size. Hahn et al. [23] further demonstrate the importance of secondary minimum deposition using colloid transport experiments conducted with different sized latex microspheres. In Hahn et al.'s study, the comparison is limited to particles ranging from $\sim 300 \mathrm{~nm}$ to $1000 \mathrm{~nm}$ in diameter. In the present work, we attempt to further our understanding of the role of particle size on colloid deposition kinetics by conducting well-controlled column deposition experiments over a broader range of particle sizes from the nano- to micro-scale.

Attachment efficiencies of the three latex particles selected in this study are considerably different over the range of IS investigated (Figure 4). The smallest nanoparticles exhibit the lowest degree of retention over the range of solution salt concentrations, whereas the two larger particles experience greater attachment to the sand surface. As indicated previously, calculations of DLVO interactions (Figure 5) suggest that the largest $(1500 \mathrm{~nm})$ colloid should experience the greatest extent of repulsion upon approach to the grain surface and therefore should exhibit the lowest degree of retention in the column experiments. However, the data presented in Figure 4 shows 
that the attachment efficiencies for the $1500 \mathrm{~nm}$ colloid are significantly greater than those of the smaller particles (at low ionic strengths). This is particularly surprising given that the height of the energy barrier is greatest at these low ionic strengths. Although the DLVO calculations imply that particles will not irreversibly attach to the quartz surface under the conditions of these experiments (i.e., in the primary energy minimum), the data does suggest that particles may be retained in the secondary energy well. In Figures $5 \mathrm{~d}-\mathrm{f}$, the interaction energy profiles are replotted to highlight the depth of the secondary energy well. The predicted depth of the secondary energy minimum $\left(\Phi_{\min }\right)$ ranges from 0.2 to $31 k_{\mathrm{B}} T$ for the largest latex particle, and from 0 to 1.6 $k_{\mathrm{B}} T$ for the smallest nanoparticle. Taken together, the calculations shown in Figure 5 and the data presented in Figure 4 suggest that particles are being retained in the secondary energy minimum. Several recent studies have also demonstrated the importance of secondary minimum deposition in the retention of colloids in granular porous media [23, 24, 42-44]. Other investigations of colloid transport in granular porous media have demonstrated the importance of physical straining as an important removal mechanism for larger colloids such as the $1500 \mathrm{~nm}$ latex microspheres used here [25, 45, 46]. Tufenkji et al [45] showed how the potential for removal by straining increases with angularity of the collector grain shape. Microscopic examination of the clean, dry sand used in our study (Figure 6) revealed grain shapes that are not always well rounded. The grain shape was analyzed further using multiple microscope images to determine the roundness factor (a parameter that describes the shape's resemblance to a circle):

$$
\text { Roundness factor }=\frac{4}{\pi} \frac{\text { Area }}{(\text { major axis })^{2}}
$$

From the equation above, a circle will have a roundness factor of 1 . Analysis of the grains used in this study yields a roundness factor of 0.67 , suggesting that grain shape may contribute to removal of particles by straining. However, in the current study, comparison of $\alpha$ values for the $110 \mathrm{~nm}$ 
colloids with those of the $1500 \mathrm{~nm}$ colloids (measured at higher ionic strengths) indicate that the smaller particles generally exhibit greater or nearly equal removal in the packed bed. This observation suggests that physical straining is not the key mechanism controlling removal of particles in the experimental system as the larger particles would exhibit significantly greater removal rates if this were the case.

\section{[FIGURE 6]}

Rather, the data obtained with the three different-sized latex colloids supports the notion of secondary minimum deposition (particularly for the larger particles) and combined primary/secondary minimum deposition for the smallest nanoparticles examined.

While the role of the secondary minimum in colloid deposition is still much debated, it is theoretically possible provided that the force resultant from the particle's kinetic energy and the fluid drag is insufficient to drive the particles out of the secondary minima. A recent experimental study using a parallel-plate flow cell packed with glass beads provides insight on the role of secondary minimum deposition on particle removal in granular porous matrices [47]. These researchers report on the direct observation of secondary minimum deposition of latex microspheres onto the surface of the glass bead collectors. Specifically, Kuznar and Elimelech suggest that at moderate ionic strength, they observe particles becoming trapped in the secondary energy well and translating along the collector surface before accumulating near the rear of the spherical collectors [47].

Calculations of the theoretical collision efficiency assume that both particles and collectors are spherical. While both the particles and collectors selected for this investigation are likely to be considerably more spherical than the majority of those encountered in realistic situations, neither the latex particles nor the sand grains are perfectly spherical. A theoretical investigation 
of the effect of surface roughness on colloid stability showed that the height of the energy barrier was considerably reduced in the presence of surface asperities on the collector surface [22]. While it was concluded that the presence of surface roughness alone could not account for the observed discrepancies with respect to particle size, it was demonstrated that surface roughness could at least be contributing to the observed erroneous particle size effect [22]. It is important to note that the effect of interfacial dynamics or deposition in secondary energy minima that were discussed above may have considerable interplay with the impact of surface roughness. However, the understanding of these factors and particularly of their interplay is still quite limited, and as such, their impact on colloidal stability cannot yet be fully assessed quantitatively [22].

\subsubsection{Effect of NOM}

The presence of natural organic matter in groundwater is an environmental factor that is often not accounted for in colloid transport studies. While it is recognized that these ubiquitous macromolecules have an important effect on soil and aquatic systems due to their role in buffering $\mathrm{pH}$ and in increasing the cation exchange capacity [27], their influence on the stability of colloidal suspensions is largely uncertain. In the second part of this study, we examine whether the presence of NOM similarly impacts the transport and deposition behavior of nanoparticles as it does that of micron-scale colloids. Colloid transport experiments were repeated with all three latex colloids in the presence of $5 \mathrm{mg} / \mathrm{L}$ SRHA and calculated attachment efficiencies are presented in Figure 7. For comparison, the data obtained in the absence of SRHA is also included.

[FIGURE 7] 
The shapes of the stability curves measured in the presence of $5 \mathrm{mg} / \mathrm{L}$ SRHA (Figure 7) are similar to those measured in the absence of the humic acids; i.e., the attachment efficiency increases with IS (at low IS). In general, the attachment efficiency of the latex colloids in the presence of NOM is lower or equal to that measured in the absence of humics. By and large, this result is in qualitative agreement with other studies investigating the influence of NOM on colloid deposition kinetics [12, 14, 38, 48, 49]. Franchi and O'Melia [12] found that SRHA adsorbed on the surface of $98 \mathrm{~nm}$ sized sulphate latex colloids reduced the deposition of the particles onto the surface of glass bead collectors and enhanced the reentrainment of deposited particles in the porous media. Similarly, adsorption of a soil humic acid onto hematite colloids caused a near 2 orders of magnitude decrease in the particle deposition rate in a sandy soil matrix [14]. Davis et al. [50] noted that the presence of $\mathrm{Ca}^{2+}$ played an important role in the influence of NOM on the deposition of sulphate functionalized latex microspheres. In the presence of calcium, addition of humic acid resulted in decreased particle deposition rates. However, in the absence of $\mathrm{Ca}^{2+}$, addition of humic acid did not have a comparable effect [50]. In a related study [49], researchers demonstrated that adsorption of humic acid onto the surface of kaolinite colloids caused a decrease in particle deposition rates. The generally observed increase in colloid stability in the presence of humics has traditionally been attributed to the effect of NOM on particle surface potential. Several researchers have shown that NOM acts to increase the absolute value of colloid zeta potential for negatively charged colloids $[12,37,38,49]$. The measurements for the two smaller nanoparticles $(50 \mathrm{~nm}$ and $110 \mathrm{~nm}$ colloids) presented in Figure 2 show such behaviour; namely, the zeta potential is considerably more negative in the presence of SRHA. Based on DLVO theory, the observed increases in the absolute value of zeta potential for these nanoparticles will give rise to increased particle stability and indeed, this has been confirmed by a corresponding decrease in $\alpha$ (Figures 7a 
and $b)$. In contrast, the zeta potential of the largest particle $(1500 \mathrm{~nm})$ is considerably less negative when $5 \mathrm{mg} / \mathrm{L}$ SRHA is added to the suspension (Figure 2). Despite this contrary and unexpected behaviour, observation of the results presented in Figure 7c shows a decrease in $\alpha$ in the presence of NOM, as was the case of the two smaller colloids. This observation is of particular interest because it suggests that surface potential effects can not fully explain the differences in attachment efficiency that were observed in the presence and absence of NOM for all three particles. The data also shows that the influence of NOM on particle surface properties is different for the nano-sized and micron-sized colloids examined here (i.e., in the presence of NOM, the nanoparticles exhibit an increase in absolute zeta potential whereas the micron-sized colloids exhibit a decrease in absolute zeta potential). However, it can be noted that the NOM effect on particle deposition is similar for all three particle sizes; namely, the degree of change in $\alpha$ is comparable for all three particles examined. Taken together, the results in Figures 2 and 7 indicate that there may be some other mechanism(s) not considered here contributing to the attachment behaviour of the colloids.

To verify whether a physical mechanism may have contributed to the observed effect of SRHA on colloid retention, the diameters of the colloids were measured and compared to those determined in the absence of NOM (Figure 1). The data shows no significant effect of NOM on particle size, suggesting that physical straining is not responsible for the larger attachment efficiencies observed for all three colloids in the absence of SRHA and that a physicochemical mechanism bears further consideration.

A possible explanation for the unusual behaviour observed in Figure $7 \mathrm{c}$ is the influence of non-DLVO forces. For example, adsorbed layers of SRHA on the surface of the particles can enhance the stability of colloids by an effect known as "steric stabilization" [16]. Hence, the observed decrease in attachment efficiency in the presence of SRHA may be a result of the 
combined effect of steric stabilization and charge stabilization. This could explain how the presence of SRHA caused a decrease in $\alpha$ for all three particle sizes irrespective of the observed effect on particle zeta potential. Indeed, several other researchers have suggested that adsorption of humic acids onto colloid surfaces may prevent deposition by a mechanism of steric stabilization $[12,13,37,51]$. The results presented here provide additional support for the proposed mechanisms of electrostatic and steric interactions that stabilize colloidal suspensions in the presence of NOM.

\section{Conclusions}

A series of colloid filtration experiments were performed using three different sized latex particles (50, 110 and $1500 \mathrm{~nm})$ across a broad range of physicochemical conditions in both the absence and presence of $5.0 \mathrm{mg} / \mathrm{L}$ SRHA. At low IS $(1-10 \mathrm{mM} \mathrm{KCl})$, an increase in attachment efficiency with increasing particle size was observed, which is in contrast with theoretical predictions of particle deposition based on retention solely in the primary energy minimum of the DLVO interaction energy profile. Calculations of DVLO interaction energies suggest that the larger particles are retained in the secondary energy minimum, whereas the smallest particles can be retained in both primary and secondary energy wells.

The addition of NOM in the form of SRHA to the experimental system resulted in a decrease in attachment efficiency for all three particle sizes. The degree of change in $\alpha$ in the presence of $5 \mathrm{mg} / \mathrm{L}$ SRHA was similar for all three particle sizes. The presence of SRHA led to an increase in the absolute zeta potential of the suspensions for the $50 \mathrm{~nm}$ and $110 \mathrm{~nm}$ colloids, but a decrease in the absolute zeta potential for the $1500 \mathrm{~nm}$ colloids. Measurements of particle size and attachment efficiencies in the presence and absence of NOM indicate that physical straining is not the key mechanism controlling particle deposition. Rather, the results suggest that 
the observed decrease in colloid attachment in the presence of NOM is related to the combined influence of the mechanisms of charge stabilization and steric stabilization. These results can have important implications for the prediction of the migration potential of engineered nanoparticles as the mechanisms identified are common to colloidal interactions in natural and engineered aquatic systems. In an effort to improve our ability to predict the transport and fate of "nano-litter" in the environment, ongoing studies are being conducted in our laboratory to compare the transport and deposition behavior of the selected nano- and micro-scale latex particles to that of engineered nanomaterials (e.g., quantum dots and metal oxides). 


\section{Acknowledgements}

This research was supported by NSERC (Discovery Grant), the Canada Research Chairs (CRC) Program, the Canada Foundation for Innovation (CFI), the Fonds québécois de la recherche sur la nature et les technologies (FQRNT Nouveaux Chercheurs) and the Eugenie Ulmer Lamothe (EUL)

Fund at McGill University. The authors thank I. A. Eydelnant (McGill) for assistance with characterization of the granular media. 


\section{References}

1. M.R. Wiesner and J.-Y. Bottero, eds. Environmental Nanotechnology: Applications and Impacts of Nanomaterials. 2007, McGraw-Hill: New York.

2. $\quad$ S. Mize, Nanotechnology Opportunity Report. 2002, CMP Cientifica.

3. H. Shand, The Big Down: From Genomes to Atoms. 2003, ETC Group: Winnipeg, Manitoba.

4. G. Jia, H. Wang, L. Yan, X. Wang, R. Pei, T. Yan, Y. Zhao, and X. Guo, Environ. Sci. Technol., 39 (2005) 1378-1383.

5. $\quad$ D.Y. Lyon, L.K. Adams, J.C. Falkner, and P.J.J. Alvarez, Environ. Sci. Technol., 40 (2006) 43604366.

6. S. Kang, M. Pinault, L.D. Pfefferle, and M. Elimelech, Langmuir, 23 (2007) 8670-8673.

7. N. Saleh, K. Sirk, Y.-Q. Liu, T. Phenrat, B. Dufour, K. Matyjaszewski, R.D. Tilton, and G.V. Lowry, Environ. Eng. Sci., 24 (2007) 45-57.

8. B. Schrick, B.W. Hydutsky, J.L. Blough, and T.E. Mallouk, Chem. Mater., 16 (2004) 2187-2193.

9. G.C.C. Yang, H.-C. Tu, and C.-H. Hung, Separation and Purification Technology, 58 (2007) 166172.

10. S.R. Kanel, D. Nepal, B. Manning, and H. Choi, Journal of Nanoparticle Research, 9 (2007) 725735.

11. H.F. Lecoanet, J.-Y. Bottero, and M.R. Wiesner, Environ. Sci. Technol., 38 (2004) 5164-5169.

12. A. Franchi and C.R. O'Melia, Environ. Sci. Technol., 37 (2003) 1122-1129.

13. S.R. Deshiikan, E. Eschenazi, and K.D. Papadopoulos, Coll. Surf. A., 145 (1998) 93-100.

14. R. Kretzschmar and H. Sticher, Environ. Sci. Technol., 31 (1997) 3497-3504.

15. K.M. Yao, M.T. Habibian, and C.R. O'Melia, Environ. Sci. Technol., 5 (1971) 1105-1112.

16. M. Elimelech, J. Gregory, X. Jia, and R.A. Williams, Particle Deposition and Aggregation:

Measurement, Modelling, and Simulation. Colloid and surface engineering series. 1995, Oxford,

England: Butterworth-Heinemann. 441.

17. N. Tufenkji and M. Elimelech, Environ. Sci. Technol., 38 (2004) 529-536.

18. B.V. Derjaguin and L.D. Landau, Acta Physicochim. URSS, 14 (1941) 733-762.

19. E.J.W. Verwey and J.T.G. Overbeek, Theory of the Stability of Lyophobic Colloids. 1948, Amsterdam: Elsevier.

20. E. Ruckenstein and D.C. Prieve, J. Chem. Soc. Faraday Trans. II, 69 (1973) 1522-1536.

21. L.A. Spielman and S.K. Friedlander, J. Coll. Interf. Sci., 46 (1974) 22-31.

22. M. Elimelech and C.R. O'Melia, Langmuir, 6 (1990) 1153-1163.

23. M.W. Hahn, D. Abadzic, and C.R. O'Melia, Environ. Sci. Technol., 38 (2004) 5915-5924.

24. G.M. Litton and T.M. Olson, Colloids and Surfaces A-Physicochemical and Engineering Aspects, 107 (1996) 273-283.

25. S.A. Bradford, S.R. Yates, M. Bettahar, and J. Simunek, Wat. Resour. Res., 38 (2002) 1327-1338.

26. M. Elimelech, Wat. Res., 26 (1992) 1-8.

27. E.M. Thurman, Organic Geochemistry of Natural Waters. 1985, Dordrecht: Springer. 497.

28. D.G. Kinniburgh, C.J. Milne, M.F. Benedetti, J.P. Pinheiro, J. Filius, L.K. Koopal, and W.H. van Riemsdijk, Environ. Sci. Technol., 30 (1996) 1687-1698.

29. G.M. Litton and T.M. Olson, Environ. Sci. Technol., 27 (1993) 185-193.

30. J.H. Masliyah and S. Bhattacharjee, Electrokinetic and Colloid Transport Phenomena. 2006: WileyInterscience. 363.

31. R.J. Hunter, Foundations of Colloid Science. 2001, New York: Oxford University Press. 806. 
32. M. Elimelech and C.R. O'Melia, Colloids and Surfaces, 44 (1990) 165-178.

33. C.F. Zukoski and D.A. Saville, J. Coll. Interf. Sci., 114 (1986) 45-53.

34. C.F. Zukoski and D.A. Saville, J. Coll. Interf. Sci., 114 (1986) 32-44.

35. A.G. van der Put and B.H. Bijsterbosch, J. Coll. Interf. Sci., 92 (1983) 499-507.

36. M. Borkovec, S.H. Behrens, and M. Semmler, Langmuir, 16 (2000) 5209-5212.

37. A. Amirbahman and T.M. Olson, Environ. Sci. Technol., 27 (1993) 2807-2813.

38. X. Dai and R.M. Hozalski, Environ. Sci. Technol., 37 (2003) 1037-1042.

39. J.A. Davis, Geochmica et Cosmochimica Acta, 46 (1982) 2381-2393.

40. J. Gregory, J. Coll. Interf. Sci., 83 (1981) 138-145.

41. R. Hogg, T.W. Healy, and D.W. Fuerstenau, Trans. Faraday Soc., 62 (1966) 1638-1651.

42. J.A. Redman, S.L. Walker, and M. Elimelech, Environ. Sci. Technol., 38 (2004) 1777-1785.

43. N. Tufenkji and M. Elimelech, Langmuir, 21 (2005) 841-852.

44. M.W. Hahn and C.R. O'Melia, Environ. Sci. Technol., 38 (2004) 210-220.

45. N. Tufenkji, G.F. Miller, J.N. Ryan, R.W. Harvey, and M. Elimelech, Environ. Sci. Technol., 38 (2004) 5932-5938.

46. J.W.A. Foppen, M. van Herwerden, and J.F. Schijven, Journal of Contaminant Hydrology, 93 (2007) 236-254.

47. Z.A. Kuznar and M. Elimelech, Coll. Surf. A., 294 (2007) 156-162.

48. W.P. Johnson and B.E. Logan, Wat. Res., 30 (1995) 923-931.

49. R.A. Akbour, J. Douch, M. Hamdani, and P. Schmitz, J. Coll. Interf. Sci., 253 (2002) 1-8.

50. C.J. Davis, E. Eschenazi, and K.D. Papadopoulos, Colloid Polym Sci, 280 (2002) 52-58.

51. A. Amirbahman and T.M. Olson, Colloids and Surfaces a-Physicochemical and Engineering Aspects, 95 (1995) 249-259. 


\section{Figure Captions}

Fig. 1. Average hydrodynamic diameter of sulphate latex colloids suspended in $\mathrm{KCl}$ in the absence (open symbols) and presence (closed symbols) of $5 \mathrm{mg} / \mathrm{L}$ SRHA at $\mathrm{pH} 5.7 \pm 0.2$.

Fig. 2. Average zeta potential of sulphate latex colloids suspended in $\mathrm{KCl}$ in the absence (open symbols) and presence (closed symbols) of $5 \mathrm{mg} / \mathrm{L}$ SRHA at $\mathrm{pH} 5.7 \pm 0.2$. (a) $50 \mathrm{~nm}$ particles, (b) $110 \mathrm{~nm}$ colloids, and (c) $1500 \mathrm{~nm}$ colloids.

Fig. 3. Representative particle breakthrough curves for experiments conducted with $1500 \mathrm{~nm}$ colloids in columns packed with clean quartz sand in the absence of NOM over a wide range of solution ionic strengths $(\mathrm{KCl})$. The key experimental conditions were as follows: approach velocity $2.12 \times 10^{-2} \mathrm{~cm} / \mathrm{s}$, porosity 0.38 , mean grain diameter $0.256 \mathrm{~mm}, \mathrm{pH}$ $5.7 \pm 0.2$, and temperature $20-22^{\circ} \mathrm{C}$.

Fig. 4. Attachment efficiencies $(\alpha)$ calculated using eq 1 for the $(\diamond) 50 \mathrm{~nm},(\square) 110 \mathrm{~nm}$, and $(\circ)$ $1500 \mathrm{~nm}$ colloids suspended in $\mathrm{KCl}$ in the absence of $\mathrm{NOM}$ over a wide range of solution ionic strengths ( $\mathrm{pH} 5.7 \pm 0.2)$. Other experimental conditions are the same as in Figure 3. Dotted lines are included as eye guides.

Fig. 5. Calculated DLVO interaction energy plotted as a function of separation distance between the grain surface and the latex particle for a range of solution ionic strengths in the absence 
of NOM (pH 5.7 \pm 0.2 ). (a) and (d) for the $50 \mathrm{~nm}$ colloid; (b) and (e) for the $110 \mathrm{~nm}$ colloid; and (c) and (f) for the $1500 \mathrm{~nm}$ colloid.

Fig. 6. Microscope images of the sand grains used in the laboratory-scale colloid transport experiments (4× magnification, $100 \mu \mathrm{m}$ bar).

Fig. 7. Attachment efficiencies ( $\alpha$ ) calculated using eq 1 for (a) $50 \mathrm{~nm}$, (b) $110 \mathrm{~nm}$, and (c) 1500 $\mathrm{nm}$ colloids suspended in $\mathrm{KCl}$ in the absence (open symbols) and presence (closed symbols) of $5 \mathrm{mg} / \mathrm{L}$ SRHA over a wide range of solution ionic strengths ( $\mathrm{pH} 5.7 \pm 0.2$ ). Other experimental conditions are the same as in Figure 3. 


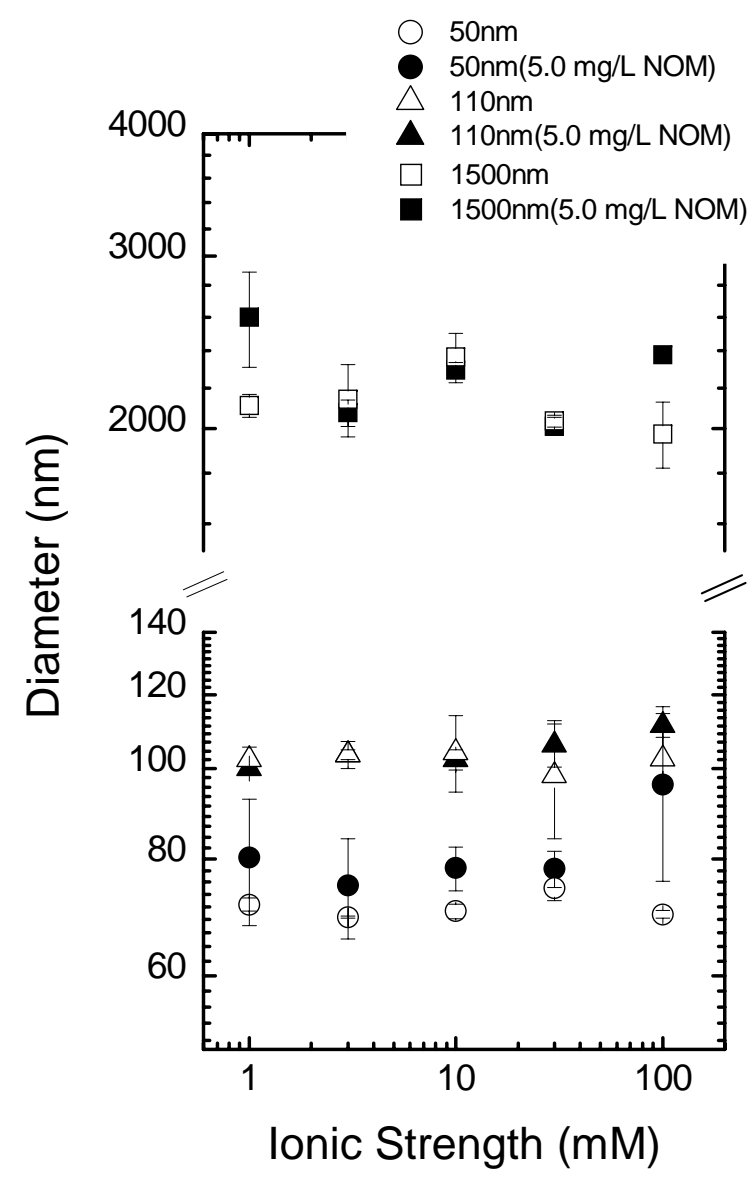



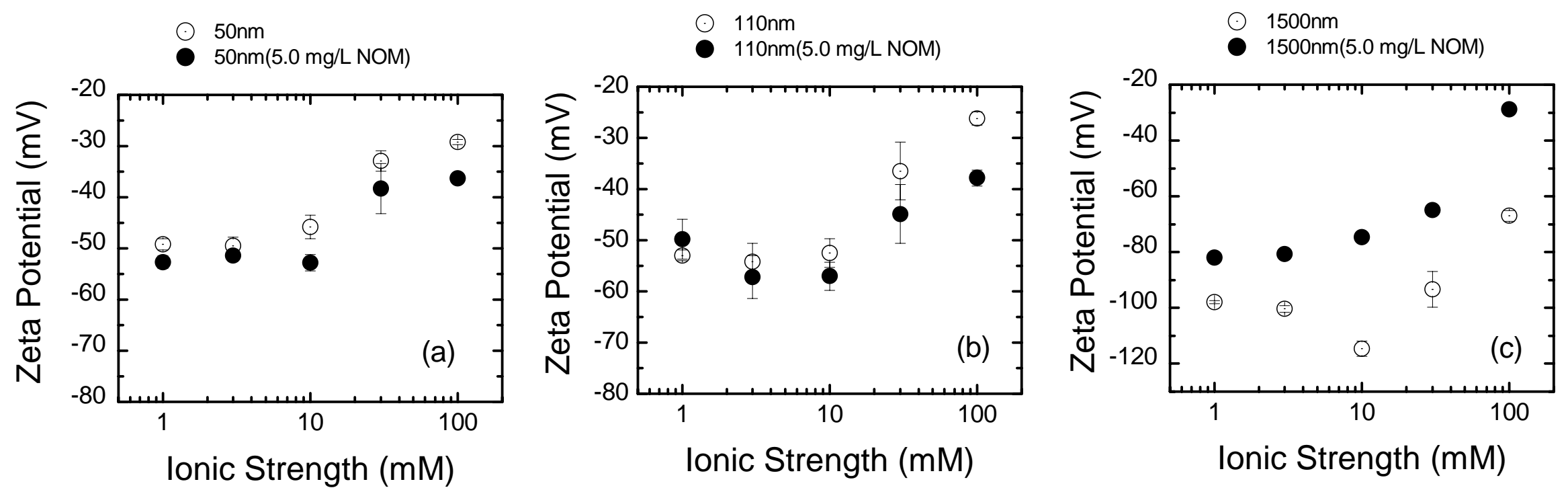


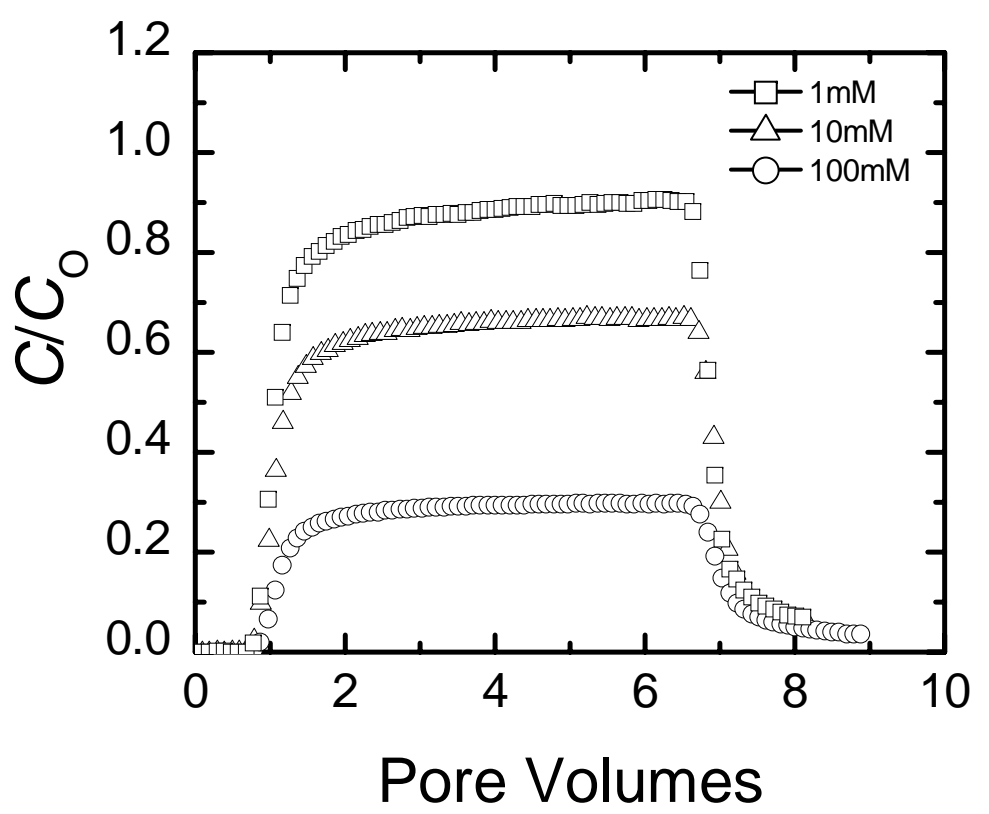




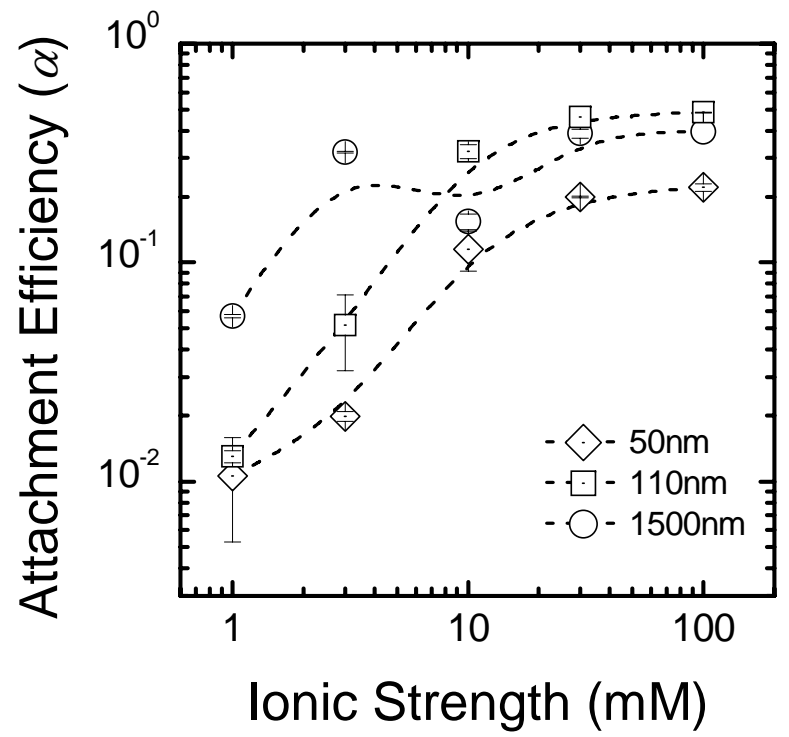



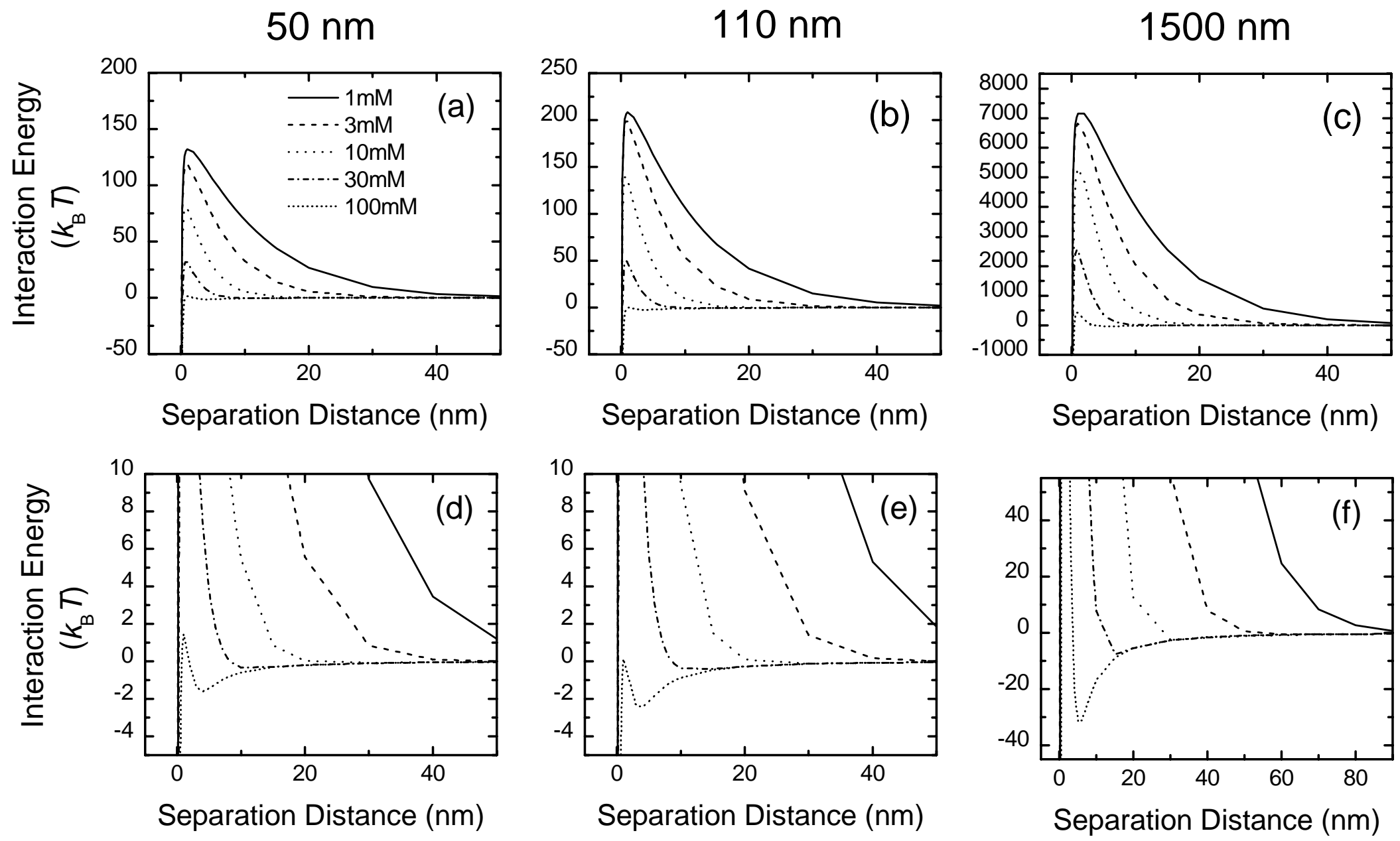


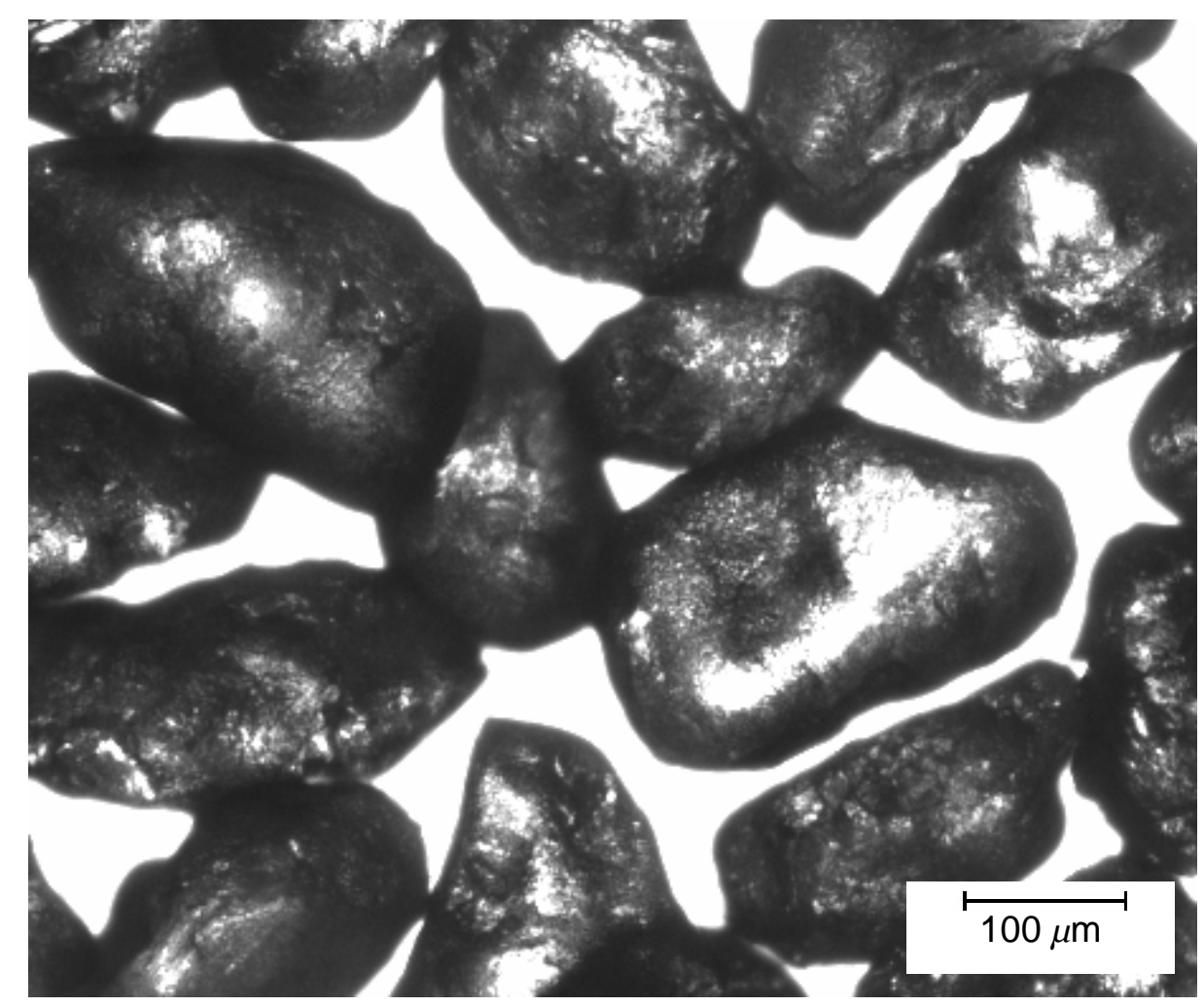



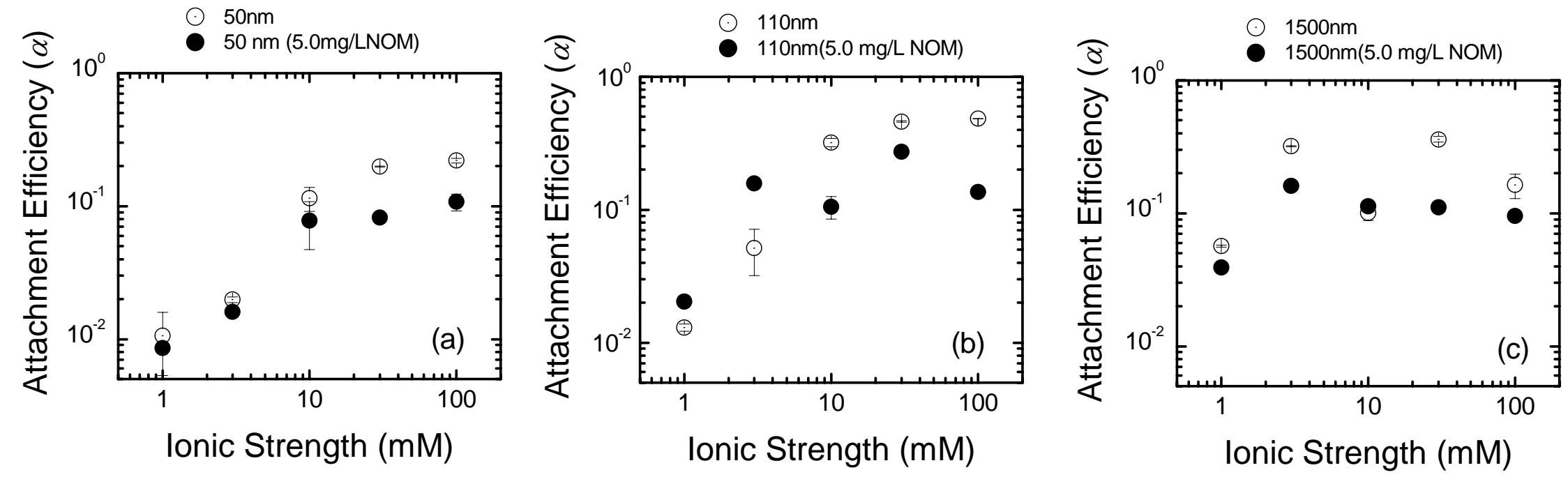